\title{
Interactions in Aqueous Solution of a Zwitterionic Surfactant with a Water Treatment Protein from Moringa oleifera Seeds Studied by Surface Tension and Ultrasonic Velocity Measurements*
}

\author{
Habauka M. Kwaambwa ${ }^{1 \#}$, Fiona M. Nermark ${ }^{2}$ \\ ${ }^{1}$ School of Health and Applied Sciences, Polytechnic of Namibia, Windhoek, Namibia \\ ${ }^{2}$ Department of Chemistry, University of Botswana, Gaborone, Botswana \\ Email: "hkwaambwa@polytechnic.edu.na, fselato@gmail.com
}

Received August 26, 2013; revised September 26, 2013; accepted October 2, 2013

Copyright (C) 2013 Habauka M. Kwaambwa, Fiona M. Nermark. This is an open access article distributed under the Creative Commons Attribution License, which permits unrestricted use, distribution, and reproduction in any medium, provided the original work is properly cited.

\begin{abstract}
The interaction of a zwitterionic surfactant with the water treatment protein extracted from Moringa oleifera has been investigated by surface tension and ultrasonic velocity measurements. The critical micelle concentration (CMC) of zwitterionic surfactant was determined to be $2.4 \pm 0.3 \mathrm{mM}$ by both techniques and the partial specific volume $v=0.78 \pm$ $0.06 \mathrm{~cm}^{3} / \mathrm{g}$ for the protein was found. There seems to be a mild interaction between the protein and the surfactant as shown by surface tension measurements. The ultrasonic velocity was found to decrease in the vicinity of the critical micelle concentration which may be due to micelle aggregates formation and the protein caused a shift of the surfactant's $\mathrm{CMC}$ to a higher concentration.
\end{abstract}

Keywords: Critical Micelle Concentration; Density; Surface Tension; Ultrasonic Velocity; Zwitterionic Surfactant

\section{Introduction}

It is well-known that solution environment such as $\mathrm{pH}$, salt and surfactant can significantly alter the behaviour and overall performance of biomolecules. In our earlier research of the protein from Moringa oleifera (MO) seeds, we have used a number of techniques to study their effects on the properties of the protein in order to understand the nature and mechanism of this protein in water treatment [1-7]. Most of these studies have used anionic surfactants particularly sodium dodecyl sulphate (SDS) although some of them used also cationic and nonanionic surfactants but not with zwitterionic surfactant. Generally, zwitterionic surfactants have been much less studied than other classes of surfactants. Their behavior is often described in relation to ionic and nonionic surfactants but the electrically neutral nature of zwitterionic surfactants clearly distinguishes them from ionic surfac-

\footnotetext{
* Competing interests: the authors declare that no competing interests exist.

${ }^{\#}$ Corresponding author.
}

tants. Studies of the MO protein-surfactant interactions have been performed in our labs aiming to understand how the surfactant binding may affect the protein structure and function and hence its efficient use for water treatment.

Zwitterionic surfactants, which contain both positively and negatively charged head groups, are interesting molecules because of their many unique properties. In general, they are mild to the skin and eyes, exhibit low toxicity, display excellent water solubility, broad isoelectric ranges, high foam stability, and resistance to hard water and to degradation by oxidizing agents [8]. Also, changes in temperature, $\mathrm{pH}$, and added electrolyte have been found to have minimal effect on zwitterionic surfactants. In order to obtain better insight into their interactions with aqueous MO protein systems, we report here the results of an investigation of the interactions of a zwitterionic surfactant with a water treatment protein extracted from MO seeds, studied by surface tension and ultrasonic velocity techniques. 


\section{Materials and Methods}

\subsection{Materials}

A zwitterionic surfactant $N$-dodecyl- $N, N$-dimethyl-3-ammonio-1-propanesulfonate (DDAPS) (also named $n$-dodecyl sulfobetaine) was supplied by Sigma Aldrich ( $\geq 98 \%$ assay, critical micelle concentration of $2-4 \mathrm{mM}$ in the range $20-25^{\circ} \mathrm{C}$, Mol. Wt. $=335.55 \mathrm{~g} \cdot \mathrm{mol}^{-1}$ ) and was used without any further purification. The structure formula of DDAPS is shown in Figure 1. Sodium chloride ( $99 \%$ purity) was supplied by Rochelle Chemicals. Distilled water was used for all experiments.

\subsection{Extraction and Purification of Protein}

The extraction and purification of protein powder was done using the method of Ndabigengesere and Narasiah [9], and the experimental details are as described previously by Kwaambwa and Maikokera [1]. To minimize the number of components, no buffer was used. This is with the view that the protein investigated is used for water treatment and buffer conditions would not reflect the environment is it intended. Furthermore, previous studies have shown that the protein is insensitive up to as high as $\mathrm{pH} 9[2,4,7]$ and the isoelectric has been reported to be between $\mathrm{pH} 10$ and 11 [9], suggesting that it is very stable and to a large extent considered self-buffering.

\subsection{Surface Tension Measurements}

Surface tension of a series of differing concentrations of surfactant (DDAPS) in the absence and presence of the freeze dried protein, were measured with a Krüss K9 tensiometer using the Du Nouy platinum ring detachment method which was calibrated using distilled water. Readings were taken in triplicates. The platinum ring was cleaned before each measurement by heating on a Bunsen burner flame to remove any residual deposits.

A series of different concentrations of surfactant were prepared from the stock solution by dilution using distilled water and varying protein concentrations in distilled water as required. The surface tension measurements were measured at room temperature immediately after sample preparation. Surfactant solutions in the presence of the protein were used to study the protein-surfactant interactions.

The surface tension data was analysed using the simplest form of the Gibbs equation written as

$$
-d \gamma=R T \sum_{i} \Gamma_{i} d \ln a_{i}
$$

where $\gamma$ is the surface tension, $R$ is the universal gas constant, and $T$ is the temperature in kelvins $(\mathrm{K})$, whereas $\Gamma_{\mathrm{i}}$ and $a_{i}$ are the surface excesses and activities of all the independent components. For measurements on a non-

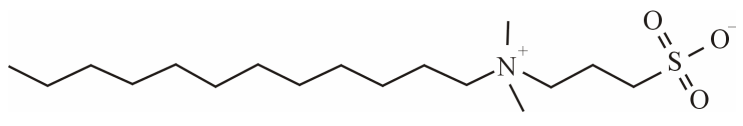

Figure 1. Schematic structure of N-Dodecyl-N,N-dimethyl3-ammonio-1-propane sulphonate, showing the location of positive and negative charges.

ionic surfactant solution, this generally reduces to

$$
-d \gamma=R T \Gamma \ln a
$$

There are some considerations concerning the definition of $\Gamma$ but these are generally unimportant in the context of surfactant systems. For nonionic surfactants, the usual practice is to use the approximation of concentrations (c) rather than activities except at or above the CMC. The slope of the plot of $\gamma$ against $\ln c$ gives the corresponding surface excess $\Gamma$ at that point using Equation (2).

\subsection{Density Measurements}

For a macromolecule such as a polyelectrolyte, a detailed understanding of the solution requires information on a variety of physical properties such as partial specific volume. The partial specific volume of a protein (or other macromolecule) is needed for a number of physicochemical techniques such as analytical ultracentrifugation, sedimentation equilibrium, and small-angle scattering. Specific volumes may be obtained from density measurements. However, partial specific volumes are not always accurately determined because experimental precision may be limited by the preparation of solutions of different concentrations. In this study, density data of the solutions were needed in the ultrasonic velocity measurements.

The measurements were performed at a constant temperature of $298.15 \mathrm{~K}$ using an Anton Parr DMA 4500 vibrating-tube precision density meter. The measurements were taken in triplicates to get an average value. The density meter was calibrated using distilled water as its density is accurately known from literature.

\subsection{Ultrasonic Velocity Measurements}

All measurements were performed at $298.15 \mathrm{~K}$. The speed of sound was measured with uncertainty of $\pm 0.3 \%$ using a multi frequency ultrasonic interferometer (Mittal Enterprises, New Delhi, India), operating at $1 \mathrm{MHz}$, which was calibrated with water. Since the speed of sound of our samples spans an interval ranging approximately from 1500 to $1525 \mathrm{~ms}^{-1}$, the apparatus was calibrated with pure water for which there was reliable speed of sound values in the literature. The temperature stability was maintained within $\pm 0.2 \mathrm{~K}$ by circulating thermostated water around the cell with a circulating pump.

Ultrasonic waves of known frequency are produced by 
a quartz crystal fixed at the bottom of the ultrasonic cell. These waves are reflected by a movable metallic plate kept parallel to the quartz crystal and standing waves are formed in the medium. This acoustic resonance gives rise to an electrical reaction on the generator driving the quartz crystal.

If the distance between crystal and plate is now increased or decreased and the variation is exactly one half wavelength, $\lambda / 2$, or multiple of it, anode current becomes maximum or minimum. In order to minimize the uncertainty of the measurement, their number $n$ is counted. Each maximum is recorded with the highest swing of the needle on the micrometer scale. The total distance, $d$, moved by the reflector is given by

$$
d=n \lambda / 2
$$

where $\lambda$ is the wavelength. A linear plot of $d$ versus $n$ gives a slope of $\lambda / 2$. The frequency of the cell crystal $v$ being accurately known ( $1 \mathrm{MHz})$, the speed of sound, $u$, is calculated by using the relation:

$$
u=\lambda v
$$

\section{Results and Discussion}

\subsection{Surface Tension Measurements}

Figure 2 shows the plot of $\gamma$ as a function of $\ln$ [DDAPS] with and without the protein based on Gibbs equation. This method is widely used to determine the critical micelle concentration (CMC) on a wide variety of surfactants [10]. The CMC of DDAPS was found to be $2.6 \pm$ $0.3 \mathrm{mM}$, which fall within the range given by the chemical supplier (i.e. 2 - $4 \mathrm{mM}$ ) and also reported in literature $[8,11]$. The shapes of the plots around the CMC show that the surfactant is impure. A pure surfactant will have a net break at the CMC but the plots do not show a net break but rather it reduces and then increases steadily, that is, it has a minimum which results from the impurity.

The effect of the protein extracted from protein on the

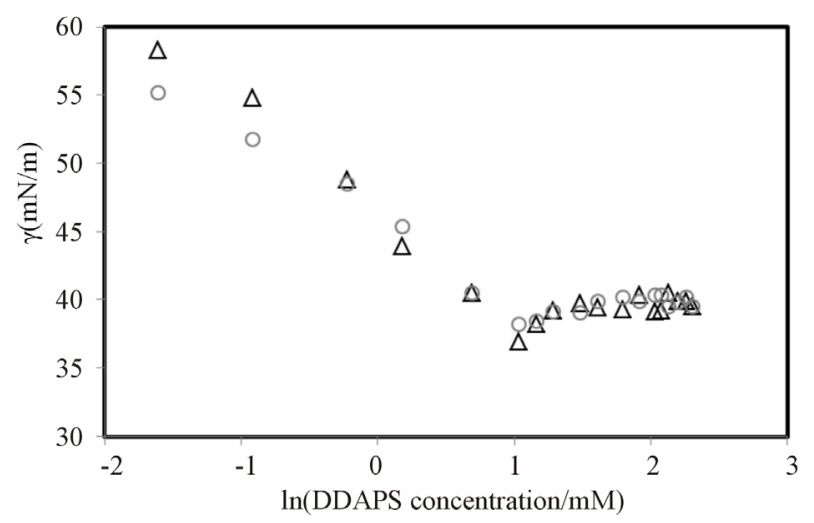

Figure 2. Variation of the surface tension a zwitterionic surfactant, DDAPS, in the presence (circles) and absence (triangles) of $0.02 \%$ MO protein. zwitterionic surfactant, DDAPS, surface tension was also studied and is shown in the same figure. The results show that the protein interacts mildly with the surfactant in the concentration range studied. In other words, the protein does not interact significantly with the zwitterionic surfactant to affect its surface activity. In fact, the behaviour observed for the protein/zwitterion system is similar to what Kwaambwa and Maikokera observed for the protein and nonionic surfactant triton X-100 (TX-100) [3]. This is not surprising because zwitterionic surfactants, although have ionic groups, are overall neutral and hence behave like nonionic surfactants.

Although an analysis of the surface tension curves can, in principle, provide quantitative information on surfactant-protein interactions, practically speaking, complete interpretation of the data becomes more difficult when the protein itself is appreciably surface active as it is the case for the protein from MO seeds. The surface tension profiles were obtained both for the surfactant systems and in the presence of protein, showing the characteristic break points in mixed protein/surfactant systems. For ease of interpretation of the analysis, the experiments were done in distilled water rather than in a buffer.

In addition to the typical CMC observed for DDAPS solutions, the profiles of the protein/surfactant mixtures show inflection points commonly known as the critical micelle concentration in the presence of protein (CMC*). The values are determined in a similar way as $\mathrm{CMC}$, i.e. the surfactant concentration at which the surface tension reaches a minimum value. The $\mathrm{CMC}^{*}$ values for different protein concentrations are normally higher than in pure water, which can be explained by considering the amount of surfactant that is bound to the protein. In our study, surface tension at $\mathrm{CMC}^{*}$, i.e. when the protein is saturated by surfactant and free micelles start to form, reached value close to that obtained for the surfactant solution alone as shown in Figure 2. It should be noted that this value is not influenced by the protein concentration as it is envisaged that protein molecules are totally displaced from the interface when the surfactant concentration reaches the $\mathrm{CMC}^{*}$.

Addition of $\mathrm{NaCl}$ seems to lower the surface tension but not the CMC as shown in Figure 3. The addition of salt reduced the surface tension more in the low DDAPS concentration region of the plot implying that more protein molecules were adsorbed onto the air/water interface to cause a reduction in surface tension (i.e. the salt makes the protein more surface active). The protein is known to be cationic (implying that the positive charges are more than the negative charges or the positive charges of the protein are more exposed) and so addition of salt results in neutralisation of the positive ions and hence making the protein more surface active. 


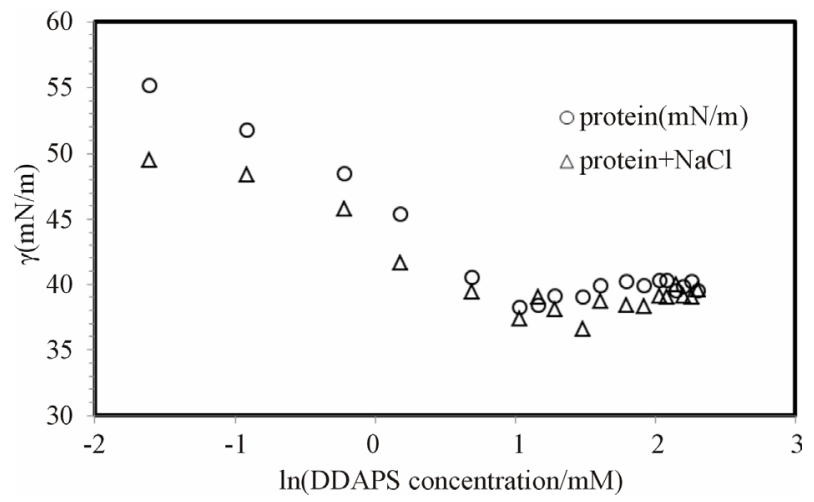

Figure 3. Variation of the surface tension of a zwitterionic surfactant, DDAPS, in $0.02 \%$ protein (circles) and in a mixture of $0.02 \%$ protein and $0.01 \mathrm{M} \mathrm{NaCl}$ (triangles).

\subsection{Density Measurements}

Figure 4 shows the linear variation of density of DDAPS as its function of concentration in the presence and absence of the protein. The partial specific volume of DDAPS was estimated from density measurements. Density values, $\rho$, increase with the concentration of the polyelectrolyte. If the partial specific volume $v$ is independent of the concentration, the following equation is obeyed

$$
\rho=\rho_{o}+\left(1-v \rho_{o}\right) c
$$

where $\rho_{o}$ is the water density and $\mathrm{c}$ is the polymer concentration in $\mathrm{g} / \mathrm{cm}^{3}$.

The dependence of the density on the concentration is linear with statistical correlation parameter, $\mathrm{R}^{2}$, greater than 0.99 and Equation (5) was applied to the data to obtain $v$ from the slope. The data yielded a partial specific volume $v=0.78 \pm 0.06 \mathrm{~cm}^{3} / \mathrm{g}$ for the protein. Experimental values for the partial specific volume of native proteins in aqueous solution cluster around 0.74 $\mathrm{cm}^{3} / \mathrm{g}$ [12]. The value in water obtained for the protein agrees well with the literature although the method used for preparing solutions of different concentrations had the limitation of not knowing precisely the protein concentration.

The protein increases the density of the DDAPS proportionally. There is no break in the plot at the CMC (determined to $2.6 \mathrm{mM}$ ) hence showing that the micellisation does not affect the density. The slopes gave the same value of partial specific volume of $v=1.0027 \pm$ $0.0002 \mathrm{~cm}^{3} / \mathrm{g}$ since the linear plots of the density versus DDAPS concentration in the presence and absence of protein are parallel in the concentration range studied (see Figure 4).

\subsection{Ultrasonic Velocity Measurements}

Figure 5 shows the plots of the ultrasonic velocities of DDAPS surfactant with and without protein. The plot of
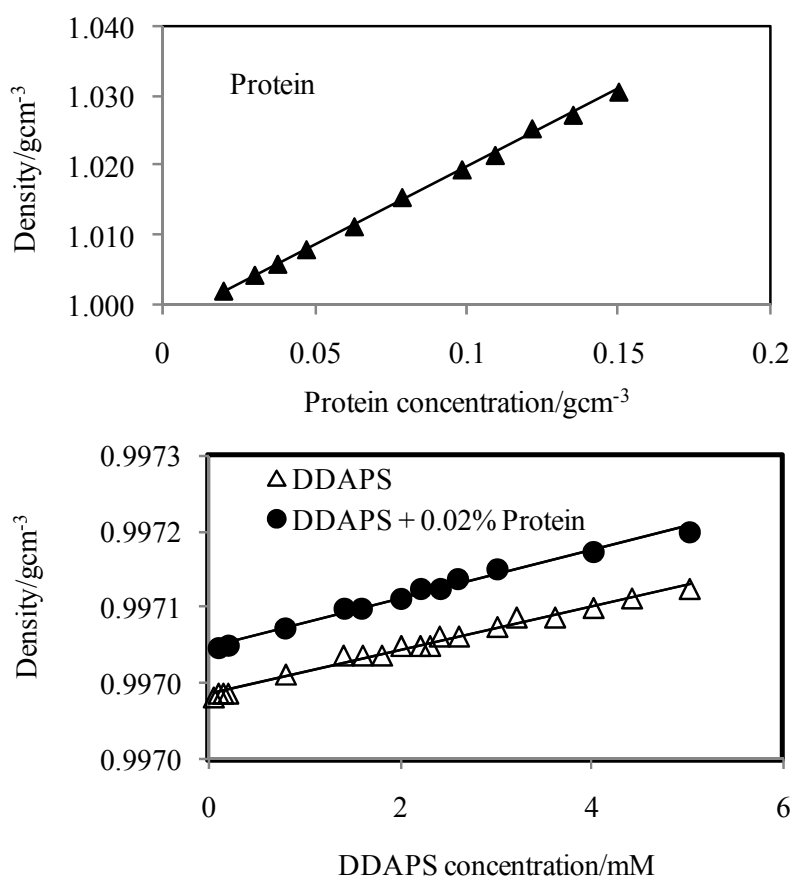

Figure 4. Variation of density of DDAPS as a function of concentration in the presence and absence protein.

surfactant with protein deviates from that without the protein but the ultrasonic velocity initially increases with increase in surfactant concentration and reaches a maximum before beginning to go down to a minimum value. After the minimum, the ultrasonic velocity begins to increase again. The minima occurred at DDAPS concentrations of $2.3 \mathrm{mM}$ and $3.0 \mathrm{mM}$ in the absence and presence of MO protein, respectively. The value for minimum for DDAPS in the absence of protein corresponds to CMC value determined from the surface tension measurements. In fact, the minimum for the plot of the surfactant with protein of $3.0 \mathrm{mM}$ falls within the range for DDAPS reported by the supplier. The MO protein causes an increase in the CMC of the surfactant which could be due to the interference of micelle aggregates formed at the CMC.

In this study, density measurements showed to increase with increase in concentration, as shown in Figure 4 , and speed of sound is said to increase with increase in density. This trend changes towards the CMC showing a decrease in the speed of sound and then an increase after the CMC. The break in the ultrasonic velocity as a function of surfactant concentration plot shows that the CMC can be determined using this technique. This technique was counterchecked using sodium dodecyl sulphate (SDS) and the results are shown in Figure 6. The ultrasonic velocity behaviour is the same as that of DDAPS and the inflection point minimum occurs at $8.3 \mathrm{mM}$, which corresponds with the reported literature $\mathrm{CMC}$ of SDS of $8.25 \mathrm{mM}$ at $25^{\circ} \mathrm{C}$ [13]. 


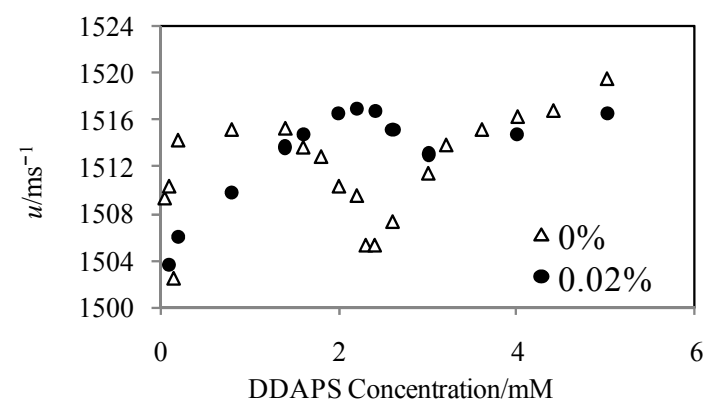

Figure 5. Ultrasonic velocity of the surfactant DDAPS in the presence $(0.02 \%)$ and absence $(0 \%)$ of the protein.

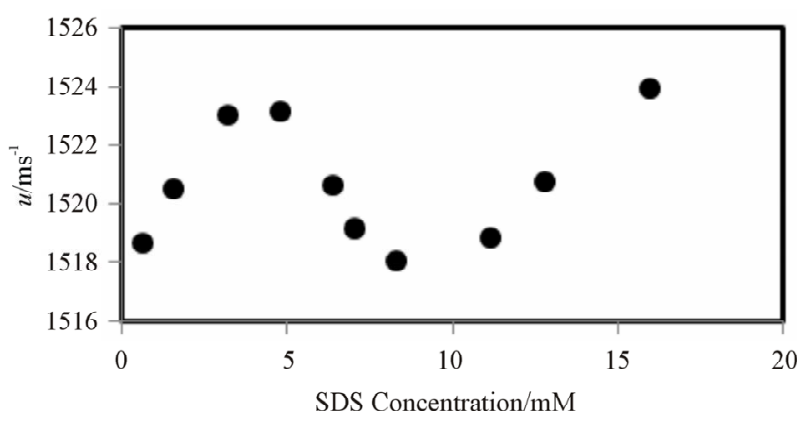

Figure 6. Ultrasonic velocity of anionic surfactant SDS as a function of concentration.

\section{Conclusion}

The zwitterionic surfactant DDAPS interacts mildly with cationic protein extracted from MO seeds as evidenced from both surface tension and ultrasonic velocity measurements. The ultrasonic velocity technique seems to be more sensitive to micellisation. The interaction behaviour of the protein with zwitterionic surfactant is similar to that observed with nonionic surfactants. The protein increases the CMC of the surfactant when investigated by ultrasonic velocity. There is a reasonable agreement in the CMC values determined by the two techniques and average result was $2.4 \pm 0.3 \mathrm{mM}$. Formation of micelles causes reduction of ultrasonic velocity through surfactant solutions whereas the protein increases proportionally the density of the DDAPS solutions with a linear relationship with surfactant concentration. The protein extracted from MO seeds slightly increases the CMC of the surfactant. There is minimum effect of $\mathrm{pH}$ on the interaction of the protein with surfactant.

\section{Acknowledgements}

The authors are grateful to the Polytechnic of Namibia IRPC and University of Botswana ORD for supporting this study.

\section{REFERENCES}

[1] R. Maikokera and H. M. Kwaambwa, "Interfacial Proper- ties and Fluorescence of a Coagulating Protein Extracted from Moringa oleifera Seeds and Its Interaction with Sodium Dodecyl Sulphate," Colloids \& Surfaces B: Biointerfaces Journal, Vol. 55, No. 2, 2007, pp. 173-178. http://dx.doi.org/10.1016/j.colsurfb.2006.11.029

[2] H. M. Kwaambwa and R. Maikokera, "A Fluorescence Spectroscopic Study of a Coagulating Protein Extracted from Moringa oleifera Seeds," Colloids \& Surfaces B: Biointerfaces Journal, Vol. 60, No. 2, 2007, pp. 213-220. http://dx.doi.org/10.1016/j.colsurfb.2007.06.015

[3] H. M. Kwaambwa and R. Maikokera, "Air-Water Interface Interaction of Anionic, Cationic, and Non-Ionic Surfactants with a Coagulant Protein Extracted from Moringa oleifera Seeds Studied Using Surface Tension Probe," Water SA, Vol. 33, No. 4, pp. 583-588.

[4] H. M. Kwaambwa and R. Maikokera, "Infrared and Circular Dichroism Spectroscopic Characterisation of Secondary Structure Components of a Water Treatment Coagulant Protein Extracted from Moringa oleifera Seeds," Colloids \& Surfaces B: Biointerfaces Journal, Vol. 64, No. 1, 2008, pp. 118-125. http://dx.doi.org/10.1016/j.colsurfb.2008.01.014

[5] R. Maikokera and H. M. Kwaambwa, "Use of Viscosity to Probe The Interaction of Anionic Surfactants with a Coagulant Protein from Moringa oleifera Seeds," Research Letters in Physical Chemistry, Vol. 2009, 2009, Article ID: 927329, 5 Pages.

[6] H. M. Kwaambwa, M. Hellsing and A. R. Rennie, "Adsorption of a Water Treatment Protein from Moringa oleifera Seeds to a Silicon Oxide Surface Studied by Neutron Reflection," Langmuir, Vol. 26, No. 6, 2010, pp. 3902-3910. http://dx.doi.org/10.1021/la9031046

[7] H. M. Kwaambwa and A. R. Rennie, "Interactions of Surfactants with a Water Treatment Protein from Moringa oleifera Seeds in Solution Studied by Zeta Potential And Light Scattering Measurements," Biopolymers, Vol. 97, No. 4, 2012, pp. 209-218. http://dx.doi.org/10.1002/bip.22014

[8] D. G. Marangoni and A. A. McLachlan, "Interactions between Zwitterionic and Conventional Anionic and Cationic Surfactants," Journal of Colloid and Interface Science, Vol. 295, No. 1, 2006, pp. 243-248. http://dx.doi.org/10.1016/i.jcis.2005.08.008

[9] A. Ndabigengesere, K. S. Narasiah and B. G. Talbot, "Active Agents and Mechanism of Coagulation of Turbid Waters Using Moringa oleifera," Water Research, Vol. 29, No. 2, 1995, pp. 703-710. http://dx.doi.org/10.1016/0043-1354(94)00161-Y

[10] P. X. Li, Z. X. Li, H.-H. Shen, R. K. Thomas, J. Penfold and J. R. Lu, "Application of the Gibbs Equation to the Adsorption of Nonionic Surfactants and Polymers at the Air-Water Interface: Comparison with Surface Excesses Determined Directly Using Neutron Reflectivity," Langmuir, Vol. 29, No. 30, 2013, pp .9324-9334.

[11] A. Amin-Alami., N. Kamenka and S. Partyka, "Interfacial Properties of Zwitterionic Surfactants: N-Dodecylbetaine," Thermochimica Act, Vol. 122, No. 1, 1987, pp. 171-179. http://dx.doi.org/10.1016/0040-6031(87)80117-X 
[12] L. R. Murphy, N. Matubayasi, V. A. Payne and R. M. Levy, "Protein Hydration and Unfolding-Insights from Experimental Partial Specific Volumes and Unfolded Protein Models," Folding \& Design, Vol. 3, No. 2, 1998, pp. 105-118.

http://dx.doi.org/10.1016/S1359-0278(98)00016-9
[13] E. A. El-Hefian and A. H. Yahaya, "Investigation on Some Properties of SDS Solutions," Australian Journal of Basic and Applied Sciences, Vol. 5, No. 7, 2011, pp. 1221-1227. 